\title{
Results and open questions on some invariants measuring the dynamical complexity of a map
}

by

\author{
Jaume Llibre and Radu Saghin (Barcelona)
}

\begin{abstract}
Let $f$ be a continuous map on a compact connected Riemannian manifold $M$. There are several ways to measure the dynamical complexity of $f$ and we discuss some of them. This survey contains some results and open questions about relationships between the topological entropy of $f$, the volume growth of $f$, the rate of growth of periodic points of $f$, some invariants related to exterior powers of the derivative of $f$, and several invariants measuring the topological complexity of $f$ : the degree (for the case when the manifold is orientable), the spectral radius of the map induced by $f$ on the homology of $M$, the fundamental-group entropy, the asymptotic Lefschetz number and the asymptotic Nielsen number. In general these relations depend on the smoothness of $f$. Various examples are provided.
\end{abstract}

1. Introduction. In this paper we consider various invariants that can be used to measure the dynamical complexity of a continuous (or smooth) map on a compact connected (Riemannian) manifold. We survey some relationships between these invariants and open questions related to them. We present examples for some situations encountered.

On one hand, we will consider the degree of the map, the spectral radius, the fundamental-group entropy, the asymptotic Lefschetz number and the asymptotic Nielsen number. These are algebraic topology invariants of $f$, which are homotopy invariants by definition. Consequently, for continuous maps on manifolds, they are stable under small perturbations.

On the other hand, there are invariants measuring the dynamical complexity of a map at the "local" level. Here we will consider the rate of growth of periodic points, the topological entropy, the volume growth, and some other invariants related to the growth rate of exterior powers of derivatives. These invariants are not stable in general, they can always be modified

2010 Mathematics Subject Classification: 37-02, 37B40, 37C15, 37C05.

Key words and phrases: spectral radius, degree, asymptotic Lefschetz number, fundamental-group entropy, asymptotic Nielsen number, topological entropy, volume growth, rate of growth of periodic points. 
by small $C^{0}$ perturbations, and sometimes, if the map is not structurally stable, they can be modified by small smooth perturbations. However, they are invariant under (maybe smooth) conjugacies.

The relationships between these invariants depend on the smoothness of the map and the main principle is the following: if we assume enough smoothness, then some dynamical complexity at the global level should imply at least the same dynamical complexity at the local level. This is the main idea behind Shub's entropy conjecture, and there are many results in this direction. We will mention some of them.

The converse of this is of course not true: there are maps with some dynamical complexity coming from some local dynamics, a horseshoe for example, while at the global level there may be no dynamical complexity, the map may be homotopic to the identity.

There are some good surveys on this topic, especially relating to the topological entropy: see for example [11], [17], [24], [26], [38], [46], [51], or for the holomorphic case [14]. In our paper we try to give a basic introduction to these invariants, mention several known facts about them, and provide examples and proofs of some simple results. The results are well known to the people working in the area, so our survey is intended mostly for nonexperts, and that is why the examples are chosen as simple as possible. We are aware that we leave out some other invariants measuring the complexity of the map (like metric or local entropy, Lyapunov exponents, etc.), and we do not discuss flows or other actions, nor generic situations. Also in the case of surfaces there are more results available, because one can use NielsenThurston theory for the study of the global dynamics of homeomorphisms (see for example [4], [5]), while the local invariants are sometimes better described using Pesin theory and Lyapunov exponents; however, we want to keep our survey short and easy to read, and covering all those things would probably require another survey.

The paper is organized as follows. In Section 2 we present the definitions of the notions that we consider. In Section 3 we discuss the global invariants, while in Section 4 we consider the local invariants. Finally, in Section 5 we present some known relationships between the local and global invariants that we think relevant for this survey.

2. Preliminary definitions. Let $M$ be an $m$-dimensional compact connected manifold and $f: M \rightarrow M$ a continuous map. A point $x \in M$ is a fixed point for $f$ if $f(x)=x$. A point $x \in M$ is a periodic point of $f$ of (least) period $n$ if $f^{n}(x)=x$ and $f^{k}(x) \neq x$ for $1 \leq k<n$.

We say that $f$ satisfies the hypothesis (H1) if the following holds:

(H1) The fixed points of $f^{n}$ are isolated for all positive integers $n$. 
We use this condition to define the rate of growth of periodic points, and in connection with the Lefschetz fixed point formula. There are weaker conditions under which one can use these notions, but for simplicity of presentation we restrict to this strong condition, which is however true for $C^{r}$ generic maps, $r \geq 1$. Here we say that a property is $C^{r}$ generic, $r \geq 0$, if it holds for a residual subset of $C^{r}$ maps, considered with the $C^{r}$ topology.

If $f$ satisfies the hypothesis (H1), then we denote by \# $\operatorname{Fix}\left(f^{n}\right)$ the number of fixed points of $f^{n}$ (this is the number of periodic points whose periods are divisors of $n$ ). Then one can define the rate of growth of periodic points to be the rate of growth of these numbers with respect to $n$, i.e.

$$
\operatorname{Per}^{\infty}(f)=\limsup _{n \rightarrow \infty}\left(\max \left\{\# \operatorname{Fix}\left(f^{n}\right), 1\right\}\right)^{1 / n} .
$$

The map $f$ will induce an action on the homology groups of $M$, which we denote $f_{*, k}: H_{k}(M, \mathbb{Q}) \rightarrow H_{k}(M, \mathbb{Q})$, for $k \in\{0,1, \ldots, m\}$. The spectral radius of $f_{*, k}$ is denoted $\operatorname{sp}\left(f_{*, k}\right)$ and equal to the largest modulus of an eigenvalue of the linear map $f_{*, k}$. The spectral radius of $f_{*}$ is

$$
\operatorname{sp}\left(f_{*}\right)=\max _{k=0, \ldots, m} \operatorname{sp}\left(f_{*, k}\right) .
$$

If we assume that $M$ is oriented, then the top homology group $H_{m}(M, \mathbb{Q})$ is isomorphic to $\mathbb{Q}$ and $f_{*, m}$ is just multiplication by an integer $\operatorname{deg}(f)$ called the degree of $f$. If $f$ is $C^{1}$ then the degree of $f$ is also the algebraically counted number of pre-images of a regular value $y$ of $f$, i.e. the sum $\sum_{f\left(x_{i}\right)=y} \operatorname{sign}\left(\operatorname{det} D f\left(x_{i}\right)\right)$, provided that local coordinates at all points agree with the orientation.

Fix $p \in M$ and a path $\alpha$ joining $p$ to $f(p)$. Define the endomorphism $f_{*}^{\alpha}: \pi=\pi_{1}(M, p) \rightarrow \pi$ by $f_{*}^{\alpha}(\gamma)=\alpha f(\gamma) \alpha^{-1}$. Let $\Gamma=\left\{\gamma_{1}, \ldots, \gamma_{s}\right\}$ be a set of generators of $\pi$ and define the length of an element $\gamma \in \pi$ as

$$
L(\gamma, \Gamma)=\min \left\{\sum_{j=1}^{l}\left|i_{j}\right|: \gamma=\gamma_{s_{1}}^{i_{1}} \ldots \gamma_{s_{l}}^{i_{l}}, l \geq 1,1 \leq s_{1}, \ldots, s_{l} \leq s\right\} .
$$

The fundamental-group entropy of $f$ is

$$
h_{*}(f)=\limsup _{n \rightarrow \infty} \frac{1}{n} \log \left(\max _{1 \leq i \leq s} L\left(\left(f_{*}^{\alpha}\right)^{n}\left(\gamma_{i}\right), \Gamma\right)\right) .
$$

It can be proved that $h_{*}(f)$ is well defined and independent of $\Gamma, p$ and $\alpha$; for more about this see e.g. [18] or [2] (in Bowen's paper the fundamental-group entropy is called the logarithm of the growth rate of $f_{*}$ on $\pi_{1}(M)$ ).

For every positive integer $n$ we define the $n$-Lefschetz number of $f$ as

$$
L_{n}(f)=\sum_{k=0}^{m}(-1)^{k} \operatorname{Tr}\left(f_{*, k}^{n}\right),
$$

where as usual $\operatorname{Tr}\left(f_{*, k}^{n}\right)$ denotes the trace of the linear map $f_{*, k}^{n}$. 
By the Lefschetz formula, if $f$ satisfies the hypothesis (H1), then we also have

$$
L_{n}(f)=\sum_{f^{n}(x)=x} \operatorname{ind}\left(x, f^{n}\right),
$$

where ind $\left(x, f^{n}\right)$ is the Poincaré index of the fixed point $x$ for the map $f^{n}$ (for more details see $[7,10]$ ). We call the rate of growth of these numbers the asymptotic Lefschetz number of $f$, i.e.

$$
L^{\infty}(f)=\limsup _{n \rightarrow \infty}\left(\max \left\{\left|L_{n}(f)\right|, 1\right\}\right)^{1 / n} .
$$

A good reference for the properties of $n$-Lefschetz numbers and the asymptotic Lefschetz number is also [20].

Similarly for every positive integer $n$ one has the $n$-Nielsen number of $f$, denoted by $N_{n}(f)$, defined as follows. First we consider the following equivalence relation $\sim$ on the set of fixed points of $f: x \sim y$ if and only if there exists a curve $\gamma$ joining $x$ and $y$ such that $f(\gamma)$ is homotopic to $\gamma$ relative to its endpoints. An equivalence class is called a fixed point class; it is an isolated subset of the set of fixed points of $f$, and hence its index can be defined. The number of fixed point classes with non-zero index (also called essential classes) is called the Nielsen number of $f$, denoted by $N(f)$. If we denote the Nielsen number of $f^{n}$ by $N_{n}(f)$ then the asymptotic Nielsen number of $f$ is

$$
N^{\infty}(f)=\limsup _{n \rightarrow \infty}\left(\max \left\{\left|N_{n}(f)\right|, 1\right\}\right)^{1 / n} .
$$

For more information about Nielsen numbers see for instance [21].

We will also consider the topological entropy of $f$, defined as follows. First define the metric $d_{n}$ on $M$ by

$$
d_{n}(x, y)=\max _{0 \leq i \leq n} d\left(f^{i}(x), f^{i}(y)\right), \quad \forall x, y \in M .
$$

A finite set $S$ is called $(n, \epsilon)$-separated with respect to $f$ if for any different $x, y \in S$ we have $d_{n}(x, y)>\epsilon$. The maximal cardinality of an $(n, \epsilon)$-separated set is denoted $S_{n}$. Define

$$
h(f, \epsilon)=\limsup _{n \rightarrow \infty} \frac{1}{n} \log S_{n} .
$$

Then the topological entropy of $f$ is

$$
h(f)=\lim _{\epsilon \rightarrow 0} h(f, \epsilon) .
$$

For equivalent definitions and properties of the topological entropy we refer the reader to [18].

Now suppose that there is a Riemannian metric on the manifold $M$. If $D \subset M$ is a $C^{1}$ disk inside $M$ and $f$ is at least $C^{1}$, then the volume growth 
of $D$ under $f$ is

$$
v(D, f)=\limsup _{n \rightarrow \infty} \frac{1}{n} \log \operatorname{Vol}\left(f^{n}(D)\right),
$$

where the volume $\mathrm{Vol}$ is with respect to the Riemannian metric induced on $f^{n}(D)$ by the metric on $M$. For $1 \leq r \leq \infty$, the supremum of all the volume growths over all the $C^{r}$ disks in $M$ is the r-volume growth of $f$ :

$$
v_{r}(f)=\sup _{D \subset M C^{r} \text { disk }} v(D, f) .
$$

For $1 \leq r_{1}<r_{2}$ we clearly have

$$
v_{r_{1}}(f) \geq v_{r_{2}}(f) .
$$

There are some other related invariants involving exterior powers of the derivative of $f$; we will define and discuss them in Section 4.

The degree, spectral radius and (asymptotic) Lefschetz numbers are homology invariants, they depend only on the action of the map $f$ on the homology of $M$. The (asymptotic) Nielsen numbers and the fundamental-group entropy are homotopy invariants: two maps which are homotopic have the same (asymptotic) Nielsen numbers. The topological entropy and the rate of growth of periodic points are invariant under conjugacy. The $r$-volume growth is invariant under $C^{r}$ conjugacies.

3. Relations between global invariants. A first well known fact is that the degree and the asymptotic Lefschetz number are always smaller than the spectral radius.

Proposition 1. For any continuous map $f$ on a compact manifold $M$ we have

$$
L^{\infty}(f) \leq \operatorname{sp}\left(f_{*}\right) .
$$

If $M$ is also orientable then

$$
|\operatorname{deg}(f)| \leq \operatorname{sp}\left(f_{*}\right) .
$$

The proof is easy, it follows directly from the definitions. We remark that the inequalities of Proposition 1 can be strict, and there is no general relation between the degree and the asymptotic Lefschetz number, as we will show in some examples at the end of the section.

The asymptotic Nielsen number and the fundamental-group entropy are somehow different, because they are related to the fundamental group of $M$, and not to the homology groups. For example, if the fundamental group is trivial, every closed loop is contractible, so there can be at most one Nielsen class, and also the fundamental-group entropy must be zero. On the other hand, we have the following result (for a proof see for example [18]). 
Proposition 2. If $f$ is a continuous map on a compact manifold $M$, then

$$
\log \left(\operatorname{sp}\left(f_{*, 1}\right)\right) \leq h_{*}(f) .
$$

Also for some manifolds the Nielsen numbers can be easily related to the Lefschetz numbers, as in the following theorems. A nilmanifold is the quotient of a nilpotent connected simply connected Lie group modulo a discrete uniform (cocompact) subgroup. A solvmanifold is defined similarly, with "nilpotent" replaced by "solvable". The definitions of infranilmanifold and infrasolvmanifold are slightly more complicated, but they have always finite covers consisting of nilmanifolds and solvmanifolds respectively.

TheOREM 3 (Anosov [1]). If $f$ is a continuous map on a nilmanifold $M$, then $N(f)=|L(f)|$, so $N^{\infty}(f)=L^{\infty}(f)$.

TheOREM 4 (McCord [33]). If $f$ is a continuous map on an infrasolvmanifold $M$, then $N(f) \geq|L(f)|$, so $N^{\infty}(f) \geq L^{\infty}(f)$.

Now we will give several examples to illustrate different relationships between these invariants. We start with the case when the invariants are trivial.

EXAMPLE 1. If $f$ is homologous to the identity, then

$$
|\operatorname{deg}(f)|=L^{\infty}(f)=\operatorname{sp}\left(f_{*}\right)=1 .
$$

If $f$ is also homotopic to the identity, then

$$
N^{\infty}(f)=e^{h_{*}(f)}=e^{0}=1 .
$$

We remark that there are cases where the map is homologous to the identity but not homotopic to the identity, for example $M$ can be a surface of negative Euler characteristic, and $f$ an orientation preserving homeomorphism inducing a non-trivial element in the Torelli group of outer automorphisms of the fundamental group. In such cases the fundamental-group entropy can be greater than one, while the homological invariants, the degree, the asymptotic Lefschetz number, and the spectral radius are all equal to one (see for example [2] for a version of the Plykin attractor, with homo$\operatorname{logy} /$ homotopy relative to some fixed points).

Another simple situation is when the manifold $M$ is simply connected.

EXAMPLE 2. If $M$ is simply connected, i.e. the fundamental group is trivial, then

$$
N^{\infty}(f)=e^{h_{*}(f)}=e^{0}=1 .
$$

The other invariants can be strictly greater, for example the map $f(z)=z^{2}$ on the Riemann sphere $\mathbb{S}^{2}$ has

$$
|\operatorname{deg}(f)|=L^{\infty}(f)=\operatorname{sp}\left(f_{*}\right)=2 .
$$


Proof. The first part follows easily from the remarks made before Proposition 2. However, the other invariants can be greater: the map $f(z)=z^{2}$ on $\mathbb{S}^{2}$ has clearly degree two, also $f_{*, 0}$ is multiplication by one, $H_{1}(M, \mathbb{Q})$ is trivial and $f_{*, 2}$ is multiplication by two, $\operatorname{so~} \operatorname{sp}\left(f_{*}\right)=2$ and $L_{n}(f)=2^{n}+1$, and consequently $L^{\infty}(f)=2$.

We remark that we have a similar situation every time when $M$ is a sphere $\mathbb{S}^{n}$ or a complex projective space $\mathbb{C} P^{n}$. For $\mathbb{S}^{n}$ the only non-trivial homology groups are $H_{0}(M, \mathbb{Q})$ and $H_{n}(M, \mathbb{Q})$, the (non-trivial) action induced by a continuous self-map $f$ is then multiplication by 1 and respectively $\operatorname{deg}(f)=d$ on $\mathbb{Q}$, so we have

$$
N^{\infty}(f)=e^{h_{*}(f)}=e^{0}=1,
$$

and

$$
|\operatorname{deg}(f)|=L^{\infty}(f)=\operatorname{sp}\left(f_{*}\right)=|d| .
$$

For $M=\mathbb{C} P^{n}$ we have a similar situation: $H_{i}(M, \mathbb{Q})$ is trivial if $i$ is odd and one-dimensional if $i$ is even, and the action induced by a continuous self-map $f$ is multiplication by $d^{i / 2}$ for some integer $d$ (see [9] for example), and if $d \neq 0$ then we get

$$
N^{\infty}(f)=e^{h_{*}(f)}=e^{0}=1,
$$

and

$$
|\operatorname{deg}(f)|=L^{\infty}(f)=\operatorname{sp}\left(f_{*}\right)=|d|^{n} .
$$

The next examples are for the case when $M$ is the torus. The degree can be strictly smaller than the other invariants if there is growth coming from the action on some intermediate homology group which is greater than the one coming from the top homology group.

ExAmple 3. For the cat map

$$
g(x, y)=(2 x+y, x+y)\left(\bmod \mathbb{Z}^{2}\right)
$$

on $\mathbb{R}^{2} / \mathbb{Z}^{2} \equiv \mathbb{T}^{2}$ we have

$$
\operatorname{deg}(g)=1<L^{\infty}(g)=\operatorname{sp}\left(f_{*}\right)=N^{\infty}(g)=e^{h_{*}(g)}=h(g)=\frac{3+\sqrt{5}}{2} .
$$

For the map $g \times g$ on $\mathbb{T}^{4}$ we have

$$
\begin{aligned}
\operatorname{deg}(g \times g)=1 & <e^{h_{*}(g \times g)}=\frac{3+\sqrt{5}}{2}<L^{\infty}(g \times g)=\operatorname{sp}\left((g \times g)_{*}\right) \\
& =N^{\infty}(g \times g)=h(g \times g)=\left(\frac{3+\sqrt{5}}{2}\right)^{2} .
\end{aligned}
$$


If $t(z)=z+\alpha(\bmod \mathbb{Z}), \alpha \in \mathbb{R} \backslash \mathbb{Q}$, is an irrational translation on $\mathbb{T}^{1}$, then for $g \times t$ on $\mathbb{T}^{3}$ we have

$$
\begin{aligned}
\operatorname{deg}(g \times t) & =L^{\infty}(g \times t)=N^{\infty}(g \times t)=1 \\
& <e^{h_{*}(g \times t)}=\operatorname{sp}\left((g \times t)_{*}\right)=h(g \times t)=\frac{3+\sqrt{5}}{2} .
\end{aligned}
$$

The proof is straightforward, one just has to use the fact that for the torus the $n$-Nielsen number coincides with the absolute value of the $n$ Lefschetz number, and with the number of periodic points of period $n$ if the map is hyperbolic (all the indices of periodic points are equal to 1 or $-1), \pi_{1}(M)$ coincides with $H_{1}(M, \mathbb{Z})$, and all the maps induced on homology can be explicitly computed. The topological entropy can also be computed directly from the definition, or by using the results from Section 4 .

REMARK 1. Actually one can show that for a linear endomorphism of the torus $A$, if all the eigenvalues have absolute value one, then all the invariants we defined are trivial (zero or one). Also we always have $h_{*}(A)=$ $\log \operatorname{sp}\left(A_{*, 1}\right)=\log \operatorname{sp}(A) \leq \operatorname{sp}\left(A_{*}\right)$. If there are eigenvalues $\lambda_{1}, \ldots, \lambda_{i}$ with absolute value strictly greater than one, we have

$$
\log \operatorname{sp}\left(A_{*}\right)=h(A)=v_{r}(A)=h_{1}(A)=\sum_{k=1}^{i} \log \left|\lambda_{k}\right|
$$

for $1 \leq r \leq \infty$ (see the next section for the definition of $h_{1}$ ), and

$$
\log \operatorname{sp}\left(A_{*, 1}\right)=h_{*}(A)=\max _{1 \leq k \leq i} \log \left|\lambda_{k}\right| .
$$

If there is an eigenvalue equal to one, then $N^{\infty}(A)=L^{\infty}(A)=1$. If all eigenvalues are different from one then we also have

$$
\begin{aligned}
\log N^{\infty}(A) & =\log L^{\infty}(A)=\log \operatorname{sp}\left(A_{*}\right)=h(A)=v_{r}(A)=h_{1}(A) \\
& =\sum_{k=1}^{i} \log \left|\lambda_{k}\right| .
\end{aligned}
$$

We finish with an example where the degree is strictly greater than the asymptotic Lefschetz (Nielsen) number, because in the sum of the traces there are cancellations.

EXAMPLE 4 . For the smooth map $f=f_{1} \times f_{2}$ from the product space of the Riemann sphere $\mathbb{S}^{2}$ by the circle $\mathbb{T}^{1}$, where $f_{1}(z)=z^{2}$ and $f_{2}(x)=x+\alpha$, $\alpha \in \mathbb{R} \backslash \mathbb{Q}$, is an irrational translation, we have

$$
\operatorname{deg}(f)=\operatorname{sp}\left(f_{*}\right)=2>N^{\infty}(f)=L^{\infty}(f)=e^{h_{*}(f)}=1 .
$$

Also in this case $h(f)=\log 2$. 
Proof. The degree of $f_{1}$ is two and the degree of $f_{2}$ is one, so the degree of $f$ is $\operatorname{deg}(f)=2$. All the homology groups are one-dimensional, and the action induced by $f$ is multiplication by $1,1,2,2$ respectively, so the spectral radius of $f_{*}$ is also $\operatorname{sp}\left(f_{*}\right)=2$. All the Lefschetz and Nielsen numbers of $f$ are zero because there are no periodic points, so $L^{\infty}(f)=N^{\infty}(f)=1$ (one can see this also from the cancellations in the trace formula). The map induced on the fundamental group is the identity, so the fundamental-group entropy is zero. The topological entropy of $f$ is

$$
h(f)=h\left(f_{1}\right)+h\left(f_{2}\right)=h\left(f_{1}\right)=h\left(\left.f_{1}\right|_{\{|z|=1\}}\right)=\log 2
$$

by a simple calculation.

4. Relations between local invariants. First, for $C^{r}$ maps, with $r>1$, the volume growth is greater that the topological entropy.

Theorem 5 (Newhouse [37]). If $f$ is $C^{r}$ on a compact Riemannian manifold $M$, with $r>1$, then the volume growth of $f$ is greater than its topological entropy:

$$
v_{r}(f) \geq h(f) .
$$

The proof is based on Pesin theory, and this is why the map is required to be $C^{r}$ with $r>1$. The volume growth can also be bounded from above in terms of the topological entropy.

Theorem 6 (Yomdin [49], [50]; see also [15]). Suppose that $f$ is $C^{r}$ on a compact Riemannian manifold $M$, with $r \geq 1$. Set

$$
R(f)=\lim _{n \rightarrow \infty} \frac{1}{n} \log \left(\sup _{x \in M}\left\|d f^{n} x\right\|\right) .
$$

Then

$$
v_{r}(f) \leq h(f)+\frac{m}{r} R(f) .
$$

In [50] Yomdin actually proves this estimate for the volume growth of $l$-dimensional disks, with $m$ replaced by $l$ (and this estimate is sharp). As a consequence, we get the following corollary.

Corollary 7. If $f$ is $C^{\infty}$ on a compact Riemannian manifold $M$, then

$$
v_{\infty}(f)=h(f) .
$$

We will mention examples of $C^{r}$ maps, $1 \leq r<\infty$, with volume growth strictly greater than entropy at the end of the section. We do not know if there are examples of $C^{1}$ maps with topological entropy strictly greater than volume growth.

Now we discuss some other invariants involving exterior powers of the derivatives of $f$. We assume that $f$ is at least $C^{1}$ and $M$ is a Riemannian manifold. Denote by $D f^{\wedge}(x)$ the mapping between the full exterior algebras 
of the tangent spaces $T_{x} M$ and $T_{f(x)} M$, induced by $D f(x)$, and let $\left\|D f^{\wedge}(x)\right\|$ be the operator norm induced by the Riemannian metric on $M$. Katok [27], Ruelle [41] and Krzyżewski [29] proved the following estimate for $C^{1}$ maps:

$$
h(f) \leq \log \sup _{x \in M}\left\|D f^{\wedge}(x)\right\| .
$$

This can be easily improved to

$$
h(f) \leq \frac{1}{n} \log \sup _{x \in M}\left\|D f^{n \wedge}(x)\right\|, \quad \forall n \in \mathbb{N} .
$$

Because $\sup _{x \in M}\left\|D f^{\wedge}(x)\right\|$ is an upper bound for the Jacobian of $f$ restricted to any submanifold of $M$, for every $1 \leq r \leq \infty$ we also have

$$
v_{r}(f) \leq \log \sup _{x \in M}\left\|D f^{\wedge}(x)\right\| .
$$

A better estimate involves the following invariant, introduced in [43]:

$$
h_{1}(f)=\limsup _{n \rightarrow \infty} \frac{1}{n} \log \int_{M}\left\|D f^{n \wedge}(x)\right\| d m,
$$

where $m$ is the Riemannian measure on $M$. This is independent of the Riemannian metric on $M$ and clearly does not exceed $\sup _{x \in M}\left\|D f^{\wedge}(x)\right\|$. The following theorem was proved by Przytycki [39] for diffeomorphisms and extended by Newhouse [37] to maps. The equality for $C^{\infty}$ diffeomorphisms was proved by Kozlovski [28].

THEOREM 8. If $f$ is a $C^{r}$ map on a Riemannian manifold $M$, with $r>1$, then

$$
h(f) \leq h_{1}(f) .
$$

If $f$ is a $C^{\infty}$ diffeomorphism then

$$
h(f)=h_{1}(f) .
$$

The nature of this result is similar to Theorem 5 and also bases on Pesin theory. Again, there are examples of $C^{r}$ maps, $1 \leq r<\infty$, with topological entropy strictly smaller than $h_{1}$, due to Misiurewicz and Szlenk [36]; we will mention them at the end of the section. As a consequence of the previous two theorems one finds that if $f$ is $C^{\infty}$ then $h_{1}(f)=v_{\infty}(f)$ and $h_{1}(f) \leq v_{r}(f)$ (the inequality may be strict, see Example 6). It would be of interest to know if the inequality also holds for $C^{r}$ maps.

Let $d_{n}$ be the metric on $M$ defined by (1), let $M_{n}$ be the $m$ th Hausdorff measure of $M$ with the metric $d_{n}$, and

$$
h_{2}(f)=\limsup _{n \rightarrow \infty} \frac{1}{n} \log M_{n} .
$$

An equivalent definition of this invariant using the volume of graphs is given by Gromov (see [15], [16]). In [43] it is proved that for a $C^{1}$ map we have 
$h_{1}(f) \leq h_{2}(f)$. It was hoped these invariants could be used to prove the entropy conjecture, but the examples of Misiurewicz and Szlenk showed that this is not possible.

The rate of growth of periodic points is again a bit more complicated, and it cannot be related in general to the topological entropy and the volume growth. For a $C^{1}$ map the volume growth is always finite, and for a Lipschitz map the topological entropy is finite, but the rate of growth of periodic points may be infinite for $C^{r}$ maps with $r \geq 2$.

THEOREM 9 (Kaloshin [23]). There exists an open set of $C^{r}$ diffeomorphisms, with $r \geq 2$, which contains a residual set of maps with superexponential growth of periodic points, so in this case $\operatorname{Per}^{\infty}(f)=\infty$.

Of course in such a situation the logarithm of the rate of growth of periodic points is strictly greater than both the topological entropy and the volume growth.

For Axiom A diffeomorphisms Bowen proved that the entropy is equal to the logarithm of the rate of growth of periodic points.

Theorem 10 (Bowen [3]). If $f$ is an Axiom A diffeomorphism on a compact manifold $M$, then

$$
h(f)=\log \operatorname{Per}^{\infty}(f) .
$$

On the other hand, there are maps for which the entropy and the volume growth are both strictly positive, while there are no periodic points at all.

EXAMPLE 5. The product of the cat map with an irrational translation $g \times t$ on $\mathbb{T}^{3}$ from Example 3 has

$$
\operatorname{Per}^{\infty}(g \times t)=1
$$

and for any $1 \leq r \leq \infty$,

$$
h(g \times t)=h_{1}(g \times t)=v_{r}(g \times t)=\log \sup _{x \in M}\left\|D(g \times t)^{\wedge}(x)\right\|=\log \frac{3+\sqrt{5}}{2} .
$$

Proof. There are no periodic points, so

$$
\operatorname{Per}^{\infty}(g \times t)=1
$$

A segment in some unstable manifold of $g \times t$ is expanded at a rate of $(3+\sqrt{5}) / 2$, and the map is $C^{\infty}$, so for $1 \leq r \leq \infty$ we get

$$
\log \frac{3+\sqrt{5}}{2} \leq v_{\infty}(g \times t)=h(g \times t)=h_{1}(g \times t) \leq v_{r}(g \times t) .
$$

On the other hand, we have

$$
v_{r}(g \times t) \leq \log \sup _{x \in M}\left\|D(g \times t)^{\wedge}(x)\right\|=\log \frac{3+\sqrt{5}}{2},
$$

which finishes the proof. 
The topological entropy $h(g \times t)$ can also be computed as the sum of $h(g)$ and $h(t)$, which can actually be shown to be $\log \frac{3+\sqrt{5}}{2}$ and 0 respectively. The other invariants can also be computed only from the definitions, without using the previous theorems.

Now we will sketch the examples with volume growth strictly greater than entropy and with $h_{1}$ strictly greater than entropy.

Example 6 (see [15], [49]). For any $1 \leq r<\infty$ there exists a $C^{\infty}$ diffeomorphism $f$ on $\mathbb{S}^{2}$ such that

$$
h(f)=0<v_{r}(f) .
$$

Also for any $1 \leq r<\infty$ there exists a $C^{r}$ diffeomorphism $f$ on $\mathbb{S}^{2}$ such that

$$
h(f)=0<v_{\infty}(f) .
$$

Proof. Let $f$ be a $C^{\infty}$ map on $\mathbb{S}^{2}$ which has topological entropy zero and a fixed point $p$ which is hyperbolic (this can be a double cover of the projectivization of a linear map on $\mathbb{R}^{3}$ with three different real eigenvalues). Assume that around $p$ there is a smooth chart in which $f$ has the form

$$
f(x, y)=\left(\frac{1}{\lambda} x, \lambda y\right)
$$

for some $\lambda>1$. Choose $s>0$ such that $t \mapsto t^{s} \sin (1 / t)$ is $C^{r}$ and let $\gamma$ be the $C^{r}$ curve given in the same chart by

$$
\gamma(t)=\left(t, t^{s} \sin \frac{1}{t}\right), \quad t \in[0, \epsilon],
$$

for some $\epsilon>0$. A simple calculation shows that

$$
\operatorname{Vol}\left(f^{n}(\gamma)\right) \geq C \lambda^{n / s}
$$

for some constant $C>0$, so

$$
v_{r}(f) \geq \frac{\log \lambda}{s}>0=h(f) .
$$

For the second part of the example one can just make a change of coordinates such that $\gamma$ becomes $C^{\infty}$ and $f$ becomes $C^{r}$.

We remark that one can adapt this example to prove that the inequalities in Theorem 6 are sharp. Based on the same idea we have the next example.

Example 7 (Misiurewicz and Szlenk [36]). For any $1 \leq r<\infty$ there exists a $C^{r}$ map $f$ from $[-1,1]$ to itself such that

$$
h(f)=0<h_{1}(f) .
$$

Proof. Let $f:[-1,1] \rightarrow[-1,1]$ be an odd $C^{r}$ function such that zero is a repelling fixed point, there are two other fixed points $p$ and $-p$ which are 
attracting and no other periodic points, and in a small neighborhood of 1 we have

$$
f(t)=(1-t)^{s} \sin \frac{1}{1-t},
$$

where $s>0$ is such that $f$ is $C^{r}$. The non-wandering set of $f$ consists of three points, so the topological entropy of $f$ must be zero. The manifold in our case is one-dimensional, so we have

$$
h_{1}(f)=\limsup _{n \rightarrow \infty} \frac{1}{n} \log \int_{-1}^{1}\left|\left(f^{n}\right)^{\prime}(t)\right| d t,
$$

hence $h_{1}$ corresponds to the growth of the total variation of $f^{n}$. Again, a simple calculation shows that if $f^{\prime}(0)=\lambda>1$ then

$$
h_{1}(f) \geq \frac{\log \lambda}{s}>0=h(f) .
$$

This example can be easily modified to be on the circle.

\section{Relationships between global and local invariants}

5.1. Entropy and global invariants. Now we try to explain some known relations between local and global invariants. One of the first such connections, other than the Lefschetz formula, is due to Dinaburg [8], who relates the topological entropy of the time-one map of the geodesic flow to the fundamental group of the manifold. As mentioned before, the basic idea is that the global dynamical complexity implies local dynamical complexity, and one of the strongest and classical versions of this idea is still open: it is the entropy conjecture formulated by Shub [45] (see also [24], and [12] for a slight generalization).

ConjeCture 11 (Entropy conjecture). If $f$ is a $C^{1}$ map on a compact manifold $M$, then the topological entropy is greater than or equal to the logarithm of the spectral radius of $f_{*}$ :

$$
\log \left(\operatorname{sp}\left(f_{*}\right)\right) \leq h(f) .
$$

Inequality (2) is not true for Lipschitz maps, and an example is the following (see also [45], [15]).

EXAmple 8. For the map $f(z)=2 z^{2} /\|z\|$ extended to $\mathbb{S}^{2}$ by $f(0)=0$, $f(\infty)=\infty$, we have

$$
h(f)=0<\log (\operatorname{deg}(f))=\log \left(\operatorname{sp}\left(f_{*}\right)\right)=\log \left(L^{\infty}(f)\right)=\log 2 .
$$

Proof. We have

$$
\|f(z)\|=2\|z\|,
$$

which means that zero is a source, infinity is a sink, and all the other orbits move from zero to infinity. This means that the non-wandering set of $f$ 
contains only zero and infinity. It is known that the topological entropy of $f$ is equal to the topological entropy of the restriction to the non-wandering set of $f, \mathrm{NW}(f)$, and if this is finite then it must be zero, i.e.

$$
h(f)=h\left(\left.f\right|_{\mathrm{NW}(f)}\right)=h\left(\left.f\right|_{\{0, \infty\}}\right)=0 .
$$

On the other hand, every point on $\mathbb{S}^{2}$ other than zero and infinity has two pre-images, so $\operatorname{deg}(f)=2$. Because $f_{*, 0}$ is the identity and $H_{1}\left(S^{2}, \mathbb{Q}\right)=0$, we get

$$
\log \left(\operatorname{sp}\left(f_{*}\right)\right)=\log (\operatorname{deg}(f))=\log \left(L^{\infty}(f)\right)=\log 2>0=h(f) .
$$

REMARK 2. The map in Example 8 is obviously Lipschitz, actually it is differentiable everywhere except at two points, and it can be modified to a map differentiable at infinity too, leaving only one singularity at $z=0$. It is not a homeomorphism, but there are known examples of piecewise linear homeomorphisms (see [40]).

The entropy conjecture seems to be very difficult in general, but there are many partial results, and we will mention some of them. First, a weaker version of the conjecture is known to be true if we require $f$ to be $C^{\infty}$.

TheOREM 12 (Yomdin [49]). If $f$ is $C^{\infty}$ on a compact manifold $M$, then the entropy conjecture is true:

$$
\log \left(\operatorname{sp}\left(f_{*}\right)\right) \leq h(f) .
$$

The proof is the combination of Theorem 6 and Proposition 22 below. As a consequence, the entropy conjecture is true for holomorphic maps too.

Some weaker versions of the entropy conjecture are obtained by replacing the spectral radius of $f$ by some other global invariants mentioned in Section 3. For example, the logarithm of the degree is smaller than or equal to the topological entropy for $C^{1}$ maps:

Theorem 13 (Misiurewicz and Przytycki [34]). If $f$ is $C^{1}$ on a compact oriented manifold $M$, then

$$
\log (\operatorname{deg}(f)) \leq h(f) .
$$

REMARK 3. Again this is not true for Lipschitz maps: see Example 4 where $\log \operatorname{deg}(f)>h(f)$.

Also the logarithm of the asymptotic Nielsen number is smaller than or equal to the topological entropy for $C^{0}$ maps:

Theorem 14 (Ivanov [19]; see also [22]). If $f$ is $C^{0}$ on a compact manifold $M$, then

$$
\log \left(N^{\infty}(f)\right) \leq h(f)
$$


Manning proved that the entropy is greater than or equal to the logarithm of the spectral radius of the map induced by $f$ on the first homology group.

Theorem 15 (Manning [33]). If $f$ is $C^{0}$ and $M$ is a compact manifold, then

$$
\log \left(\operatorname{sp}\left(f_{*, 1}\right)\right) \leq h(f) .
$$

Bowen extended the result of Manning to the fundamental group of $M$.

THEOREM 16 (Bowen [2]). If $f$ is $C^{0}$ and $M$ is a compact manifold, then

$$
h_{*}(f) \leq h(f) .
$$

Question 1. Is it true that $\log \left(L^{\infty}(f)\right) \leq h(f)$ for $C^{1}$ maps? (This is not true again for Lipschitz maps because of the same Example 8).

This is a weaker version of the entropy conjecture because of Proposition 1 . We remark that $L^{\infty}(f)$ can be strictly smaller than $\operatorname{sp}\left(f_{*}\right)$ as in Example 4 or the last map in Example 3.

Other versions of the entropy conjecture are obtained by adding some restrictions on the manifold $M$. In [35] Misiurewicz and Przytycki showed that the entropy conjecture is true for continuous maps on tori, and in [24] Katok proposed another version for the entropy conjecture.

Conjecture 17 (Katok's version of entropy conjecture). If $f$ is a continuous self-map on a compact manifold $M$ with universal cover homeomorphic to a Euclidean space then

$$
\log \left(\operatorname{sp}\left(f_{*}\right)\right) \leq h(f) .
$$

On one hand, this is weaker than Shub's entropy conjecture because there are some restrictions on the manifold $M$, but on the other hand it is stronger because it only requires that $f$ is continuous. In [31] Marzantowicz and Przytycki showed that the entropy conjecture is true for continuous selfmaps on nilmanifolds, and in [32] they obtained the following generalization.

Theorem 18 (Marzantowicz and Przytycki [32]). If $f$ is a continuous self-map of a compact $K(\pi, 1)$ manifold $M$ with the fundamental group $\pi$ torsion free and virtually nilpotent, then

$$
\log \left(\operatorname{sp}\left(f_{*}\right)\right) \leq h(f) .
$$

This is the situation if $M$ is an infranilmanifold for example. Also the entropy conjecture is true if $f$ is $C^{1}$ and $M$ is a sphere $\mathbb{S}^{n}$ (this is just a consequence of Theorem 13, as is mentioned in [15] for example), or if $f$ is a diffeomorphism and $\operatorname{dim} M \leq 3$ (this is a consequence of Poincaré duality and Theorems 13 and 15, as is remarked in [46] for instance). 
Another way to obtain versions of the entropy conjecture is to add some restrictions on the dynamics of $f$. Shub and Williams, and Ruelle and Sullivan, proved that the entropy conjecture is true for Axiom A diffeomorphisms satisfying the no-cycle condition.

TheOREM 19 (see [42], [48]). If $f$ is a diffeomorphism on $M$ which satisfies Axiom $A$ and the no-cycle condition, then

$$
\log \left(\operatorname{sp}\left(f_{*}\right)\right) \leq h(f) .
$$

Saghin and Xia proved that the entropy conjecture is true for partially hyperbolic diffeomorphisms with one-dimensional center.

Theorem 20 (Saghin and Xia [44]). If $f$ is a partially hyperbolic diffeomorphism on a compact manifold $M$ and the center bundle of $f$ is onedimensional, then

$$
\log \left(\operatorname{sp}\left(f_{*}\right)\right) \leq h(f) .
$$

Fried and Shub proved that the entropy conjecture also holds when the map $f$ is $C^{1}$ and has a finite chain-recurrent set.

Theorem 21 (Fried and Shub [13]). If $f$ is a $C^{1}$ diffeomorphism on a compact manifold $M$ and the chain-recurrent set of $f$ is finite, then

$$
\log \left(\operatorname{sp}\left(f_{*}\right)\right) \leq h(f) .
$$

5.2. Volume growth and global invariants. The relationship between the volume growth and the topological invariants is easier. The next proposition shows the well known fact (see for instance [49]) that for a $C^{1}$ map the volume growth is greater than or equal to the logarithm of the spectral radius (and of the degree and asymptotic Lefschetz number).

Proposition 22. If $f$ is $C^{1}$ on a compact manifold $M$ then for all $1 \leq r \leq \infty$ we have

$$
\log \left(\operatorname{sp}\left(f_{*}\right)\right) \leq v_{r}(f)
$$

and consequently

$$
\log (\operatorname{deg}(f)) \leq v_{r}(f), \quad \log \left(L^{\infty}(f)\right) \leq v_{r}(f) .
$$

For the first inequality just use the fact that the integral of a differential form over a cycle is bounded from above by the sum of the volumes of the simplexes forming the cycle (up to a constant), because the manifold is compact. The other inequalities follow from Proposition 1.

Using the relationships with the topological entropy, it is also clear that for $C^{r}$ maps with $r>1$, the volume growth is greater than or equal to the logarithm of the asymptotic Nielsen number and the fundamental-group entropy. 
QUESTION 2. What is the relationship between the volume growth of a $C^{1}$ map and the homotopy invariants, i.e. the (logarithm of the) asymptotic Nielsen number and the fundamental-group entropy?

5.3. Rate of growth of periodic points and global invariants. Now we try to relate the rate of growth of periodic points to the topological invariants. A first easy remark is the following.

Proposition 23. For any continuous map $f$ on a compact manifold $M$ we have

$$
N^{\infty}(f) \leq \operatorname{Per}^{\infty}(f) .
$$

If $f$ satisfies the hypothesis (H1) and the indices of periodic points are uniformly bounded, then

$$
L^{\infty}(f) \leq \operatorname{Per}^{\infty}(f) .
$$

If $f$ satisfies the hypothesis (H1) and the indices of periodic points are all strictly positive (or all strictly negative), then

$$
\operatorname{Per}^{\infty}(f) \leq L^{\infty}(f)
$$

The proof is again straightforward from the definitions. We remark that the condition that the indices of periodic points are uniformly bounded is a $C^{r}$ generic property, $r \geq 1$. Actually a $C^{r}$ generic map, with $r \geq 1$, has all periodic points hyperbolic, so each index is plus or minus one. Shub and Sullivan [47] proved that the index of a fixed periodic point is uniformly bounded for all the powers of a $C^{1}$ map $f$. The following natural question was posed by M. Shub in [45].

Question 3. Is it true that for a $C^{1}$ map on a compact manifold $M$ we always have

$$
L^{\infty}(f) \leq \operatorname{Per}^{\infty}(f) ?
$$

This inequality is not true for Lipschitz maps, a counterexample is again the map from Example 8, where we have only two periodic points, so the rate of growth of periodic points is one, but the asymptotic Lefschetz number is two.

The condition that all the indices of periodic points have the same sign is satisfied for example if the diffeomorphism is uniformly hyperbolic, when all the indices are one or all are minus one, or if the map is holomorphic (see $[9])$.

In general there is no relation between the rate of growth of periodic points and the other global invariants. The global invariants may be nontrivial while there may be no periodic points at all because of the existence of an irrational translation (see Examples 3 and 4). On the other hand there may be superexponential growth of periodic points while the global invariants are finite (see Theorem 9), or there may be growth of periodic 
points because of some local complexity while at the global level the map is trivial (see Example 10 below).

For the identity map all the invariants are trivial. The simplest nontrivial example where all the invariants are equal (up to taking the logarithm if necessary) is the following.

ExAmple 9. Let $f: \mathbb{T}^{1} \rightarrow \mathbb{T}^{1}, f(x)=2 x(\bmod \mathbb{Z})$. Then for every $1 \leq r \leq \infty$ we have

$$
\begin{aligned}
\log 2 & =\log \left(N^{\infty}(f)\right)=\log \left(L^{\infty}(f)\right)=\log (\operatorname{deg}(f))=\log \left(\operatorname{sp}\left(f_{*}\right)\right)=h_{*}(f) \\
& =h(f)=h_{1}(f)=h_{2}(f)=v_{r}(f)=\log \left(\operatorname{Per}^{\infty}(f)\right) .
\end{aligned}
$$

Proof. Follows from the definitions.

An example where all the global invariants are trivial while the local ones are not is the following.

EXAMPLE 10. Let $f$ be a $C^{\infty}$ map on the sphere $\mathbb{S}^{2}$ which is homotopic to the identity and has a Smale horseshoe. Then

$$
\log \left(N^{\infty}(f)\right)=\log \left(L^{\infty}(f)\right)=\log (\operatorname{deg}(f))=\log \left(\operatorname{sp}\left(f_{*}\right)\right)=h_{*}(f)=0,
$$

while for every $r \geq 1$ we have

$$
h(f), h_{1}(f), h_{2}(f), v_{r}(f), \log \left(\operatorname{Per}^{\infty}(f)\right) \geq \log 2>0 .
$$

Proof. As in Example 1, because the map is homotopic to the identity, all the global invariants must be trivial. On the other hand, there exists an invariant hyperbolic set on which the map is conjugate to the full twoshift $\sigma_{2}$, so

$$
\# \operatorname{Fix}\left(f^{n}\right) \geq \# \operatorname{Fix}\left(\sigma_{2}^{n}\right)=2^{n} .
$$

Thus the rate of growth of periodic points is at least $2, \operatorname{Per}^{\infty}(f) \geq 2$. Also we have $h(f) \geq h\left(\sigma_{2}\right)=\log 2$. The map is $C^{\infty}$, so

$$
v_{r}(f) \geq v_{\infty}(f)=h(f)=h_{1}(f)
$$

and $h_{2}(f) \geq h_{1}(f)$ and the conclusion follows.

A map on the 2-dimensional sphere homotopic to the identity and containing a Smale horseshoe can be easily constructed. Using homotopy, one can deform a small square to create a thin rectangle, and then bend it so that the rectangle will intersect the original square transversally twice, while keeping everything unchanged outside a neighborhood of the small square.

Acknowledgements. The first author has been supported by the grants CIRIT 2009SGR 410 and MEC/FEDER MTM 2008-03437. The second author has been supported by the grant SB2006-0137. The second author would also like to thank CRM Barcelona for their hospitality.

We would like to thank the referee for the valuable comments and suggestions. 


\section{References}

[1] D. V. Anosov, The Nielsen numbers of maps on nilmanifolds, Russian Math. Surveys 40 (1985), 149-150.

[2] R. Bowen, Entropy and the fundamental group, in: The Structure of Attractors in Dynamical Systems, Lecture Notes in Math. 668, Springer, Berlin 1978, 21-29.

[3] -, Topological entropy and axiom A, in: Global Analysis, Proc. Sympos. Pure Math. 14, Amer. Math. Soc., 1970, 23-41.

[4] P. Boyland, Topological methods in surface dynamics, Topology Appl. 58 (1994), 223-298.

[5] - Isotopy stability of dynamics on surfaces, in: Geometry and Topology in Dynamics (Winston-Salem, NC, 1998/San Antonio, TX, 1999), Contemp. Math. 246, Amer. Math. Soc., Providence, RI, 1999, 17-45.

[6] R. B. S. Brooks, R. F. Brown, J. Pak and D. H. Taylor, Nielsen numbers of maps of tori, Proc. Amer. Math. Soc. 52 (1975), 398-400.

[7] R. F. Brown, The Lefschetz Fixed Point Theorem, Scott, Foresman and Co., Glenview, IL, 1971.

[8] E. I. Dinaburg, A connection between various entropy characterizations of dynamical systems, Izv. Akad. Nauk SSSR Ser. Mat. 35 (1971), 324-366 (in Russian).

[9] N. Fagella and J. Llibre, Periodic points of holomorphic maps via Lefschetz numbers, Trans. Amer. Math. Soc. 352 (2000), 4711-4730.

[10] J. M. Franks, Homology and Dynamical Systems, CBMS Reg. Conf. Ser. Math. 49, Amer. Math. Soc., Providence, RI, 1982.

[11] J. Franks and M. Misiurewicz, Topological methods in dynamics, in: Handbook of Dynamical Systems, Vol. 1A, North-Holland, Amsterdam, 2002, 547-598.

[12] D. Fried, Entropy and twisted cohomology, Topology 25 (1986), 455-470.

[13] D. Fried and M. Shub, Entropy linearity and chain-reccurence, Publ. Math. IHES 50 (1979), 203-214.

[14] S. Friedland, Entropy of holomorphic and rational maps: a survey, in: Dynamics, Ergodic Theory, and Geometry, Math. Sci. Res. Inst. Publ. 54, Cambridge Univ. Press, 2007, 113-128.

[15] M. Gromov, Entropy, homology and semialgebraic geometry (after Y. Yomdin), Séminaire Bourbaki, Vol. 1985-86, exp. 663, Astérisque 145-146 (1987), 225-240.

[16] - On the entropy of holomorphic maps, Enseign. Math. 49 (2003), 217-235.

[17] —, Three remarks on the geodesic dynamics and fundamental groups, ibid. 46 (2000), 391-402.

[18] B. Hasselblatt and A. Katok, Principal structures, in: Handbook of Dynamical Systems, Vol. 1A, North-Holland, Amsterdam, 2002, 1-203.

[19] N. V. Ivanov, Entropy and the Nielsen numbers, Soviet Math. Dokl. 26 (1982), $63-66$.

[20] J. Jezierski and W. Marzantowicz, Homotopy Methods in Topological Fixed and Periodic Points Theory, Topol. Fixed Point Theory Appl. 3, Springer, 2006.

[21] B. Jiang, Lectures on Nielsen Fixed Point Theory, Contemp. Math. 14, Amer. Math. Soc., Providence, RI, 1983.

[22] - Estimation of the number of periodic orbits, Pacific J. Math. 172 (1996), 151-185.

[23] V. Y. Kaloshin, Generic diffeomorphisms with superexponential growth of number of periodic orbits, Comm. Math. Phys. 211 (2000), 253-271.

[24] A. Katok, The entropy conjecture, in: Smooth Dynamical Systems, Izdat. "Mir", Moscow, 1977, 181-203 (in Russian). 
[25] A. Katok, Lyapunov exponents, entropy and periodic orbits for diffeomorphisms, Inst. Hautes Études Sci. Publ. Math. 51 (1980), 137-173.

[26] - Fifty years of entropy in dynamics: 1958-2007, J. Modern Dynam. 1 (2007), $545-596$.

[27] —, The estimation from above for the topological entropy of a diffeomorphism, in: Global Theory of Dynamical Systems (Evanston, IL, 1979), Lecture Notes in Math. 819, Springer, Berlin, 1980, 258-264.

[28] O. S. Kozlovski, An integral formula for topological entropy of $C^{\infty}$ maps, Ergodic Theory Dynam. Systems 18 (1998), 405-424.

[29] K. Krzyżewski, Note on topological entropy, Bull. Acad. Polon. Sci. Sér. Sci. Math. Astronom. Phys. 16 (1968), 465-467.

[30] A. Manning, Topological entropy and the first homology group, in: Dynamical Systems-Warwick 1974, Lecture Notes in Math. 468, Springer, Berlin, 1975, 185-190.

[31] W. Marzantowicz and F. Przytycki, Entropy conjecture for continuous maps of nilmanifolds, Israel J. Math. 165 (2008), 349-379.

[32] —, - Estimates of the topological entropy from below for continuous self-maps on some compact manifolds, Discrete Contin. Dynam. Systems Ser. A 21 (2008), 501-512.

[33] C. K. McCord, Estimating Nielsen numbers on infrasolvmanifolds, Pacific J. Math. 154 (1992), 345-368.

[34] M. Misiurewicz and F. Przytycki, Topological entropy and degree of smooth mappings, Bull. Acad. Polon. Sci. Sér. Sci. Math. 25 (1997), 573-574.

[35] —, - Entropy conjecture for tori, ibid. 25 (1997), 575-578.

[36] M. Misiurewicz and W. Szlenk, Entropy of piecewise monotone mappings, Studia Math. 67 (1980), 45-63.

[37] S. Newhouse, Entropy and volume, Ergodic Theory Dynam. Systems 8 (1988), 283299.

[38] —, Entropy in smooth dynamical systems, in: Proc. Int. Congress of Mathematicians, Vol. I, II (Kyoto, 1990), Math. Soc. Japan, Tokyo, 1991, 1285-1294.

[39] F. Przytycki, An upper estimation for topological entropy of diffeomorphisms, Invent. Math. 59 (1980), 205-213.

[40] C. C. Pugh, On the entropy conjecture: a report on conversations among $R$. Bowen, M. Hirsch, A. Manning, C. Pugh, B. Sanderson, M. Shub and R. Williams, in: Dynamical Systems-Warwick 1974, Lecture Notes in Math. 468, Springer, Berlin, 1975, 257-261.

[41] D. Ruelle, An inequality for the entropy of differentiable maps, Bol. Soc. Brasil. Mat. 9 (1978), 83-88.

[42] D. Ruelle and D. Sullivan, Currents, flows and diffeomorphisms, Topology 14 (1975), 319-327.

[43] R. Sacksteder and M. Shub, Entropy of a differentiable map, Adv. Math. 28 (1978), 181-185.

[44] R. Saghin and Z. Xia, The entropy conjecture for partially hyperbolic diffeomorphism with 1-D center, preprint.

[45] M. Shub, Dynamical systems, filtrations and entropy, Bull. Amer. Math. Soc. 80 (1974), 27-41.

[46] -, All, most, some differentiable dynamical systems, in: International Congress of Mathematicians (Madrid, 2006), Vol. III, Eur. Math. Soc., Zurich, 2006, 99-120.

[47] M. Shub and D. Sullivan, A remark on the Lefschetz fixed point formula for differentiable maps, Topology 13 (1974), 189-191.

[48] M. Shub and R. Williams, Entropy and stability, Topology 14 (1975), 329-338. 
[49] Y. Yomdin, Volume growth and entropy, Israel J. Math. 57 (1987), 285-300.

[50] - $C^{k}$-resolution of semialgebraic mappings, Addendum to [49], ibid., 301-317.

[51] L.-S. Young, Entropy in dynamical systems, in: Entropy, Princeton Ser. Appl. Math., Princeton Univ. Press, Princeton, NJ, 2003, 313-327.

Jaume Llibre

Radu Saghin

Departament de Matematiques

Centre de Recerca Matematica

Universitat Autonoma de Barcelona

Apartat 50

Bellaterra, 08193, Spain

E-mail: jllibre@mat.uab.cat Bellaterra, 08193, Spain E-mail: rsaghin@crm.cat

Received 12 October 2008;

in revised form 17 May 2009 